\title{
Pretreatment CT texture features for prognostication in patient with Stage III Non-Small Cell Lung Cancer
}

\author{
David Vincent Fried, SL Tucker, S Zhou, ZX Liao, Geoffrey Ibbott, Laurence Court \\ Department of Radiation Physics, The University of Texas MD Anderson Cancer Center, Houston, TX, USA.
}

Received March 19, 2014; Published Online April 08, 2014

[Presented at the Young Investigator's Symposium at the 2014 Annual Meeting of

Southwest Chapter of American Association of Physicists in Medicine (AAPM) in San Antonio, Texas, USA]

\section{Conference Proceeding}

\begin{abstract}
Purpose: To determine whether CT texture features can yield prognostic information in addition to conventional prognostic factors in stage III non-small cell lung cancer (NSCLC).

Methods: We conducted a retrospective review of 91 patients with stage III NSCLC treated with definitive chemoradiation. All patients received a four-dimensional (4D) CT simulation, where we utilized the average image (average-CT) and an expiratory image (T50-CT), and a diagnostic contrast enhanced CT image (CE-CT). A penalized cox regression model was used for covariate selection and model development. Models incorporating texture features from the 3 image types and clinical factors were compared to models incorporating clinical factors alone for overall survival (OS), local-regional control (LRC), and freedom from distant metastases (FFDM). Predictive Kaplan-Meier curves were generated using leave-one-out cross-validation. Stratification into low-risk and high-risk groups was based on a patient's predicted outcome being greater or less than the median. Reproducibility of texture features was evaluated using test-retest scans from independent patients. The concordance correlation coefficient (CCC) was used to assess texture feature reproducibility and classification accuracy was used to assess reproducibility of texture features within the context of our models.
\end{abstract}

Presenting author: David Vincent Fried; Department of Radiation Physics, The University of Texas MD Anderson Cancer Center, Houston, TX, USA.

Cite this article as:

Fried DV, Tucker SL, Zhou S, Liao ZX, Ibbott GS, Court LE. Pretreatment CT texture features for prognostication in patient with Stage III Non-Small Cell Lung Cancer. Int J Cancer Ther Oncol 2014; 2(2):020223. DOI: 10.14319/ijcto.0202.23
Results: Models incorporating both texture and clinical features demonstrated a significant improvement in stratification compared to models using clinical features alone in cross-validated Kaplan-Meier curves in terms of OS ( $\mathrm{p}=$ $0.046)$, LRC ( $\mathrm{p}=0.01)$, and FFDM $(\mathrm{p}=0.005)$. The average CCC was $0.89,0.91$, and 0.67 for texture features extracted from the average-CT, T50-CT, and CE-CT, respectively. Incorporating reproducibility uncertainties within our model yielded $80.4(\mathrm{SD}=3.7), 78.3(\mathrm{SD}=4.0)$, and $78.8(\mathrm{SD}=3.9)$ percent classification accuracy for OS, LRC, and FFDM, respectively.

Conclusion: Pretreatment tumor texture may provide prognostic information in additional to routinely obtained clinical features. Reproducibility of CE-CT appears inferior to average-CT and T50-CT; however model classification accuracy rates of $\sim 80 \%$ were still achieved.

Key Results To analyze the influence of texture reproducibility on our models, we determined the mean and standard deviation of the differences between each texture feature acquired on test-retest images. We randomly sampled a normal distribution with the aforementioned mean and standard deviation for each texture feature and added this uncertainty to the matching feature in our 91 patients. A comparison of the original model versus a model incorporating feature reproducibility is shown in Figure 2. Patients that remained in the same risk category (red and green) divided by the total number of patients were used to calculate classification accuracy. 


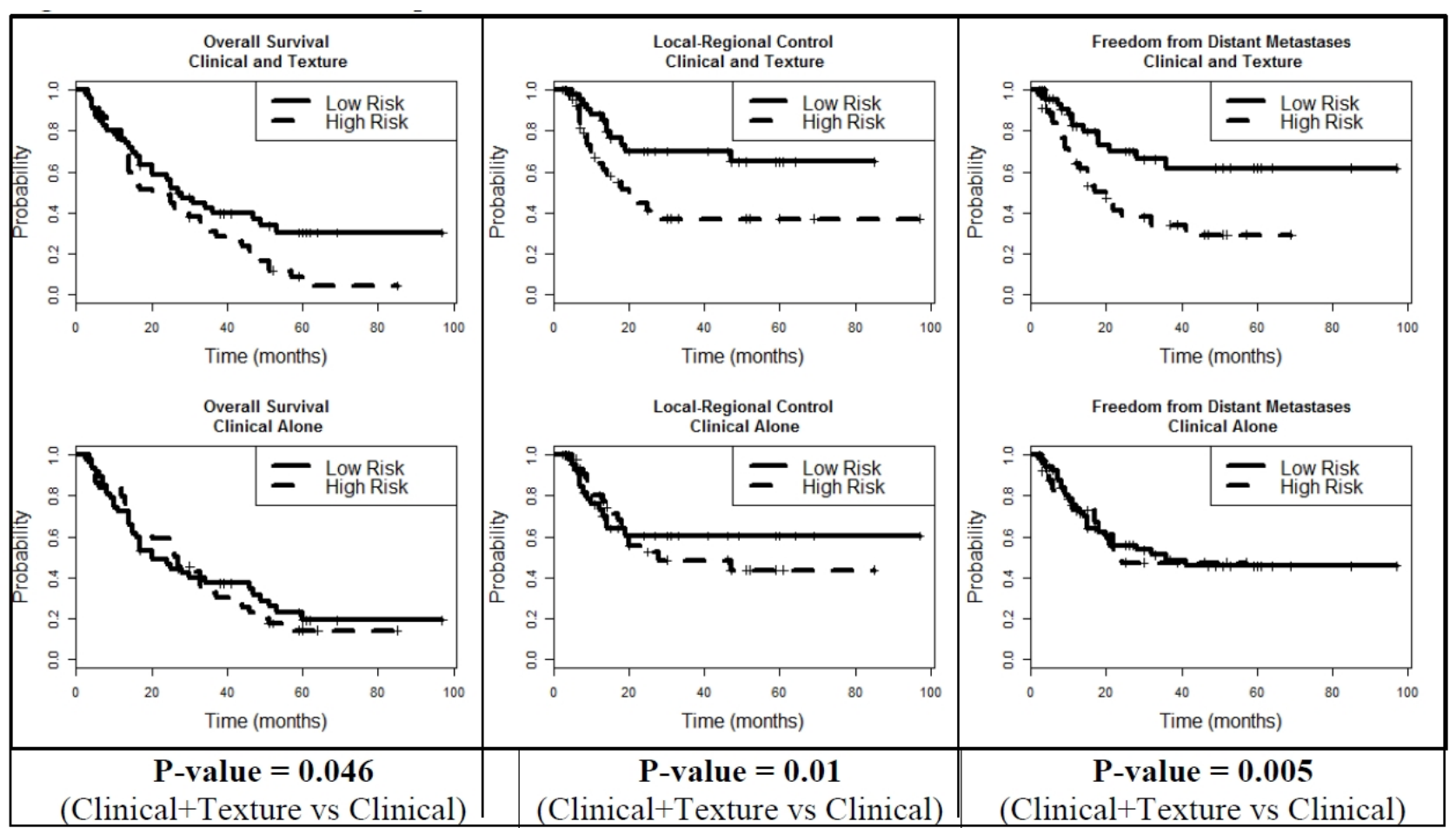

FIG. 1: Cross-validated Kaplan-Meier Curves for Models With and Without Texture Features.

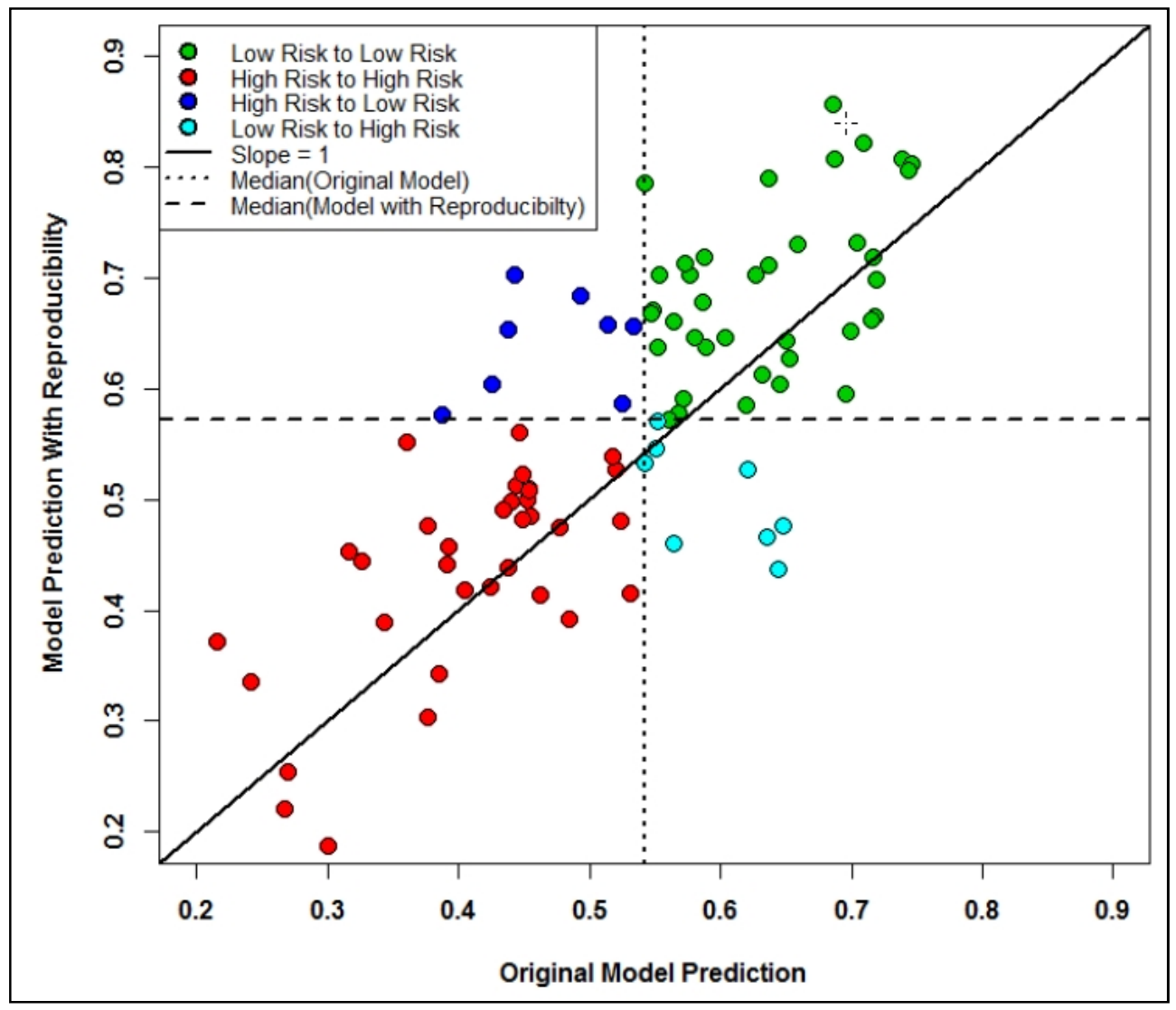

FIG. 2: Impact of Texture Feature Reproducibility on FFDM Estimates 